\title{
Revisitando Derek de Solla Price na Cientometria brasileira: análise de citações em artigos da SciELO.br
}

\author{
Revisiting Derek de Solla Price in Brazilian Scientometry: analysis of citations in SciELO.br
}

\begin{abstract}
articles
\end{abstract}
Maria Cristina Piumbato Innocentini Hayashi

Doutora em Educação pela Universidade Federal de São Carlos - UFSCar, Brasil.

Professora Titular do Departamento de Ciência da Informação e dos Programas de Pós-Graduação em Ciência, Tecnologia e Sociedade; em Educação e em Educação Especial da Universidade Federal de São Carlos - UFSCar,

Brasil.

http://orcid.org/0000-0003-1250-3767

E-mail: $\underline{\text { dmch@ufscar.br }}$

\begin{abstract}
Alexandre Masson Maroldi
Doutor em Educação pela Universidade Federal de São Carlos - UFSCar, Brasil. Professor do Curso de Biblioteconomia da Universidade Federal de Rondônia, Brasil.

http://orcid.org/0000-0002-6592-7750

E-mail: alexandre@unir.br
\end{abstract}

Carlos Roberto Massao Hayashi

Doutora em Educação pela Universidade Federal de São Carlos - UFSCar, Brasil.

Professor Associado do Departamento de Ciência da Informação da Universidade Federal de São Carlos e dos Programas de Pós-Graduação em Educação e em Ciência, Tecnologia e Sociedade da Universidade Federal de

São Carlos - UFSCar, Brasil.

http://orcid.org/0000-0003-1481-5545

E-mail: massao@ufscar.br

\section{Resumo}

Considerando a importância de Derek de Solla Price para o campo da Cientometria esse artigo objetiva traçar um panorama histórico da sua vida e representatividade de suas obras nos estudos métricos da informação, e verificar na coleção de periódicos da SciELO.br quais são os artigos com abordagens bibliométrica e cientométrica que citaram as obras de Derek Solla Price. A metodologia adotada utilizou a pesquisa bibliográfica para traçar um panorama histórico da vida e obra de Price e suas principais contribuições para os estudos métricos da informação. A fonte de dados foi a coleção de periódicos SciELO.br, e para analisar os artigos selecionados foram utilizadas a análise bibliométrica e de conteúdo. Os resultados mostraram que de um universo inicial de artigos $(\mathrm{n}=391)$ somente $6,9 \%(\mathrm{n}=27)$ citaram as obras de Price. Esses artigos foram publicados entre 1998 e 2019 em diversos periódicos ( $n=13)$, com maior incidência naqueles da área de Ciência da Informação $(n=3)$. Entre os autores $(n=49)$ predominaram aqueles do campo da Ciência da Informação $(\mathrm{n}=30)$ sendo os demais $(\mathrm{n}=19)$ de outras áreas de conhecimento $(\mathrm{n}=9)$. As obras mais citadas foram Little Science, Big Science ( $\mathrm{n}=19)$ e Networks of scientific papers $(\mathrm{n}=5)$. Os conceitos de Bibliometria, Cientometria, e Política Científica e de Lei do Elitismo e Frente de Pesquisa foram os mais utilizados nos artigos (n=14), bem como suas aplicações, respectivamente, em indicadores bibliométricos, cientométricos e de impacto, e nas análises de produção e produtividade científica e análises citações. Em conclusão, ainda há muito o que ser explorado nas obras de Price para aqueles que atuam no campo de estudos bibliométricos e cientométricos.

Palavras-chave: Derek de Solla Price. Bibliometria. Cientometria.

InCID: R. Ci. Inf. e Doc., Ribeirão Preto, v. 12, n. 1, p. 19-40, mar./ago. 2021.

DOI: 10.11606/issn.2178-2075.v12i1p19-40 


\begin{abstract}
Considering the importance of Derek de Solla Price to the field of Scientometry, this article objective to draw a historical panorama of his life and the representativeness of his works in the metric studies of information, and to verify in the collection of journals of SciELO.br which are the articles with bibliometric and scientometric approaches that cited the works of Derek Solla Price. The methodology adopted used bibliographic research to draw a historical panorama of Price's life and work and his main contributions to the metric studies of information. The data source was the collection of SciELO.br journals, and bibliometric and content analysis were used to analyze the selected articles. The results showed that of an initial universe of articles $(n=391)$ only $6.9 \%(n=27)$ cited Price's works. These articles were published between 1998 and 2019 in several journals $(n=13)$, with a greater incidence in those in Information Science $(n=3)$. Among the authors $(n=49)$, those in the field of Information Science $(n=30)$ predominated, with the others $(n=19)$ from other areas of knowledge $(n=9)$. The most cited works were Little Science, Big Science $(n=19)$ and Networks of scientific papers $(n=5)$. The concepts of Bibliometry, Scientiometrics, and Scientific Policy and the Law of Elitism and the Research Front were the most used in the articles $(n=14)$, as well as their applications, respectively, in bibliometric, cientometric and impact indicators, and in production and scientific productivity analysis and citation analysis. In conclusion, there is still much to be explored in Price's works for those who work in the field of bibliometrics and cientometrics studies.
\end{abstract}

Keywords: Derek de Solla Price. Bibliometry. Scientometry.

\title{
1. Introdução
}

Nos dias atuais as atividades relacionadas as pesquisas científicas estão constantemente sujeitas aos processos de avaliação. Nesse cenário, os estudos com abordagem bibliométrica se tornaram uma importante metodologia para avaliar o estágio de evolução de pesquisas nos mais diversos campos do conhecimento.

Galyavieva (2014, p. 220), refere que nos últimos anos o interesse pelas chamadas "métricas (bibliometria, cienciometria, informetria e outros) vem crescendo em todo o mundo". Para corroborar essa visão o autor cita o estudo de Van Noorden (2010) publicado na Nature, que revelou uma explosão das métricas, ou seja, um aumento de dez vezes no número de publicações bibliométricas nos últimos 20 anos.

São essenciais para a realização de estudos bibliométricos e cientométricos o domínio de conhecimentos teóricos de várias áreas de conhecimento, notadamente e ddisciplinas relacionadas ao campo dos Estudos Sociais da Ciência, como a Sociologia da Ciência, a História, Filosofia e Antropologia da Ciência, e outras identificadas com o campo da Ciência da Informação, por exemplo, a Comunicação Científica. Além disso, conhecimentos de Matemática e Estatísticas são mandatórios para aqueles que desejam aplicar as principais leis bibliométricas - de Lotka, de 
Bradford e de Zipf - em seus estudos, bem como elaborar e interpretar indicadores de produção científica e tecnológica.

No âmbito dos inúmeros pesquisadores dessas áreas da ciência, Derek John de Solla Price, ou apenas Price - como é mais conhecido - pode ser considerado como "a figura dominante" (KINOUCHI, 2014, p.149) pelos bibliometristas em virtude de sua extensa contribuição ao campo dos estudos métricos da informação. Hayashi (2013) assinala que apoiando-se sobre toda uma série de trabalhos anteriores, mas defendendo uma ciência da ciência, Price amplia consideravelmente a perspectiva da Bibliometria. Já Simão Mathias, que escreveu a apresentação da edição brasileira da obra Little Science, Big Science, descreve Price como um dos "mais distintos historiadores da ciência contempôranea" (MATHIAS, 1976, p.14).

Outros dois notáveis pesquisadores do campo da Sociologia da Ciência e da Cientometria, Robert Merton e Eugene Garfield, atribuem a Price o título de "pai da cientometria", uma vez que seus estudos tem um grande impacto no uso de indicadores quantitativos na formulação das políticas públicas (ADEDAYO, 2015; GARFIELD, 2009; BUCKLAND, 2017; CHAVARRO, 2020).

Nesse sentido, em um esforço para examinar a natureza altamente cumulativa da ciência, Price foi o pioneiro nesses estudos, uma vez que tinha a capacidade de resumir generalizações importantes sobre a ciência e seus padrões quantitativos simples, ou seja, através da lógica e da matemática conseguiu demonstrar, entre outros, um modelo de crescimento científico conectando o tamanho da ciência e do tempo (FERNÃNDEZ-CANO; TORRALBO; VALLEJO, 2004). Para Gingras (2016, p. 29), Price "propõe analisar a ciência tal como faz a física estatística com o gás, isto é, seguindo a evolução do conjunto dos cientistas ou de suas publicações, em vez da limitação de centrar-se num sábio em particular".

Em seu legado como pesquisador, Price deixou um conjunto de conceitos, teorias e aplicações matemáticas em seus escritos. Braga (1974) e Crawford (1984) destacam que o autor estudou o comportamento das redes de citações bibliográficas, dando nova dimensão aos estudos bibliométricos; descreveu a natureza da Ciência, da comunicação e da produtividade científica, através de leis internacionalmente aceitas: Frente de Pesquisa, Colégios Invisíveis, Crescimento Exponencial, Elitismo etc.; estabeleceu fundamentos para a política científica e tecnológica que 
têm sido largamente utilizados por vários países. Afora a atuação nesses campos de conhecimento, Braga (1974, p. 155) complementa que as pesquisas de Price são diversificadas, pois:

[...] descobriu o único manuscrito sobre Astronomia do poeta inglês Geoffrey Chaucer; estabeleceu a relação entre os relógios d'água chineses e os medievais europeus; analisou e interpretou o complexo e sofisticado mecanismo de Antikythera, um calendário astronômico descoberto em 1901 e construído aproximadamente em 80 a.C.

Apesar de grande parte de suas contribuições terem sido publicadas entre os anos de 1960 e 1980, elas ainda estão presentes na literatura científica atual, conforme mostrou o estudo de Garfield (2008), ao verificar que vinte e cinco anos após a sua morte Price ainda era citado em cerca de 100 trabalhos academicos anualmente. Estudo anterior de Garfield (1985) também havia apontado as citações de Litte Science, Big Science recebidas em 260 periódicos, o que demonstra o impacto e a influência generalizada dessa obra para o campo da Cientometria. Bonitz (1994) comenta que esse livro junto com os artigos Networks of scientific papers (PRICE, 1965) e $A$ general theory of bibliometric and other cumulative advantage process (PRICE, 1976a) continuavam a receber 70 citações por ano, sendo que três entre quatro dessas citações vinham de periódicos das Ciências Sociais. Martín-Martín; Orduna-Malea e López-Cózar (2018), em uma pesquisa de cunho bibliométrico para descrever as disciplinas acadêmicas através de citações do Google Acadêmico, detectaram que Derek de Solla Price é o quarto autor especialista nos estudos bibliométricos mais citado com 13.263 citações e Index $H$ de 33. Ao longo de sua notável carreira, Price publicou entre os anos de 1941 e 1985 quase três centenas documentos entre livros, artigos de periódicos e outros (BEDINI, 1984).

Outra comprovação de que os estudos de Derek Solla Price são relevantes para a ciência data de 1984, um ano após a sua morte prematura. Nessa ocasião, a revista Scientometrics instituiu a Derek Price de Solla Price Memorial Medal, um prêmio que até hoje é oferecido a pesquisadores com relevantes contribuições acadêmicas para o campo da cientometria (Figura 1). Erfanmanesh e Moghiseh (2019) e Mokhtarpour e Khasseh (2020) corroboram afirmando que a Derek de Solla Price Medal é o primeiro e mais importante prêmio concebido aos estudos cientométricos. Vale ressaltar que até 1989, a premiação foi anual, e após uma pausa de quatro anos, a partir de 1993, o prêmio passou a ser bianual. 
Figura 1 - Medalha Derek de Solla Price, e laureados
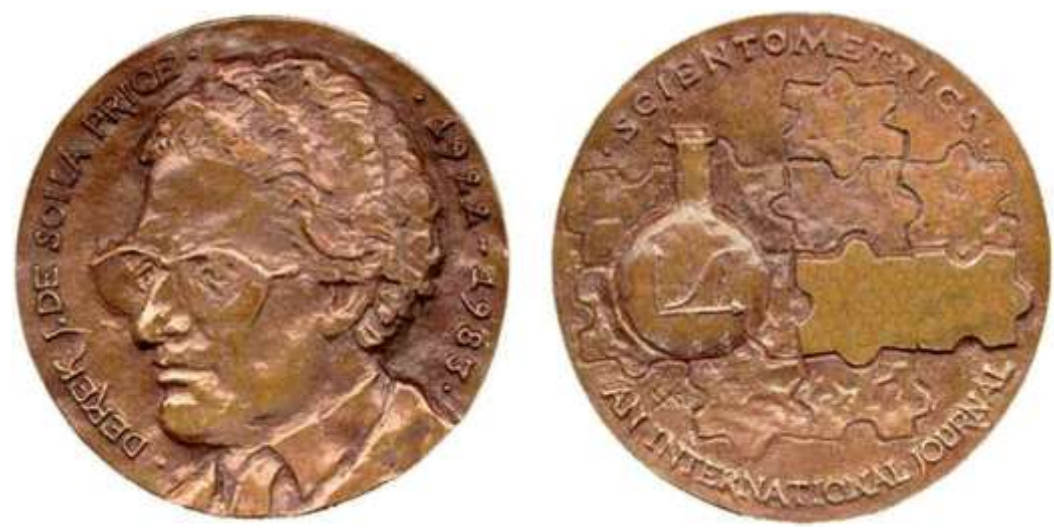

Laureados: Eugene Garfield (1984), seguido por Michael J. Moravcsik (1985), Tibor Braun (1986); Vasili V. Namilov e Henry Small (1987), Francis Narin (1988), Bertram C. Brookes e Jan Vlachy (1989), András Schubert (1993), Anthony Van Raan e Robert Merton (1995), John Irvine, Bem Martin e Belver C. Griffith (1997), Wolfgang Glänzel e Henk F. Moed (1999), Leo Egghe e Ronald Rousseau (2001), Loet Leydesdorff (2003), Peter Ingwersen e Howard D. White (2005), Katherine W. McCain (2007), Peter Vinckler e Michel Zitt (2009), Olle Persson (2011), Blaise Cronin (2013), Mike Thelwall (2015), Judith Bar-Ilan (2017), Lutz Bornmann (2019).

Fonte: Elaboração dos autores, com base em International Society for Scientometrics and Informetrics (2020).

Após esse preâmubulo e tendo em vista a importância das contribuições de Price nos estudos bibliométricos, foi realizada uma pesquisa com seguintes objetivos: traçar um panorama histórico da sua vida e representatividade de suas obras nos estudos métricos da informação, e verificar na coleção de periódicos da SciELO.br quais são os artigos com abordagens bibliométrica e cientométrica que citaram as obras de Derek Solla Price. Vale observar que por "obras" entendese os artigos, livros, capítulos e trabalhos apresentados em eventos por este autor. Para alcançar os objetivos propostos, o manuscrito se pautou na pesquisa bibliográfica e nas metodologias bibliométrica e de análise conteúdo. As próximas seções apresentam os resultados obtidos na pesquisa realizada. 


\section{A trajetória de Derek Solla Price nos estudos métricos da informação}

Para elaborar esse panorama histórico da trajetória acadêmica e científica de Derek de Solla Price com ênfase nos estudos métricos da informação foi realizado um estudo bibliográfico que compulsou suas principais obras e de outros autores da literatura científica que refletiram sobre sua influência nos campos da Cientometria e Sociologia da Ciência.

Derek John de Solla Price (1922-1983) desde criança demonstrou considerável aptidão em matemática e ciências. Obteve $P h$. D. em Física, $P h . D$. em História da Ciência, Avalon Professor de História da Ciência da Universidade de Yale, que criou para ele o Department of History of Science and Medicine. Foi ainda Consultor da National Science Foundation, VicePresidente do Internacional Council for Science Policy Studies, e detentor de inúmeros títulos e prêmios científicos em âmbitos nacional e internacional (BRAGA, 1974; WOUTERS; LEYDESDORFF, 1994). Todavia, Price percorreu um longo caminho nos meandros da ciência até ser considerado nos dias atuais como um dos mais notáveis pesquisadores relativos ao campo da Cientometria e da História da Ciência.

Para Adedayo (2015) e Yagi, Badash e Beaver (1996), Price foi um notável cientista com ampla influência principalmente nos estudos da Sociologia da Ciência. Suas explorações científicas também abarcavam as Ciências Naturais, particularmente as Ciências Físicas e Matemáticas. Sua carreira científica começou como um assistente de laboratório de Física, no entanto, seus esforços científicos culminaram na Ciência da Informação, área em que é considerado um cientista especialista para o estudo avançado em Bibliometria e Cientometria.

Price escreveu extensivamente ao longo de sua carreira expondo vários conceitos e ideias, contudo é possível considerar dois momentos distintos de sua produção científica e que afetam diretamente conteúdo de suas ideias: o primeiro até os anos de 1950 e o segundo posterior a essa data.

O primeiro doutorado de Price foi em Física teórica e experimental obtido em 1946 na Universidade de Londres e suas primeiras publicações foram nessa área de conhecimento. Dois anos depois, então 26 anos de idade, Price chega a Malásia. Naquela ocasião, leu a coleção completa do periódico Philosophical Transactions of the Royal Society of London, despertando

InCID: R. Ci. Inf. e Doc., Ribeirão Preto, v. 12, n. 1, p. 19-40, mar./ago. 2021. 
nele um novo e duradouro interesse sobre os contextos históricos da ciência. Ademais, Price também identificou como um padrão irregular nos tamanhos das pilhas de volumes quando eles eram empilhados em ordem cronológica. Propenso a determinar se tal padrão era observável em outros casos, Price coletou dados sobre o aumento anual no número de artigos indexados na Physics Abstracts e - fiel à hipótese - verificou que este crescimento era exponencial (ou seja, dependente, para uma constante extensão, no número acumulado de artigos) em vez de linear (FURNER, 2003a; YAGI, BADASHI, BEAVER, 1996; TURNER, 1984; KOCHEN, 1984).

Ao retornar à Inglaterra com seus novos interesses no ano de 1950, Price iniciou um segundo doutorado, desta vez na História da Ciência no Christ's College, em Cambridge. Price apresentou suas descobertas iniciais sobre o crescimento exponencial da literatura no $6^{\circ}$ Congresso Internacional de História da Ciência, em Amsterdã. Em agosto de 1950 seu artigo foi republicado em francês na revista Archives Internationales d'Histoires des Sciences e uma 'peça' de acompanhamento sobre material semelhante apareceu cinco anos depois, na popular revista de ciência britânica Discovery. Foi nesse momento em que o autor toma a decisão de deixar o campo da física para entrar no campo da história da ciência. Na visão de Furner (2003a), essa foi uma difícil decisão do autor, e só aconteceu pois Price considerava que o campo da história da ciência tanto na Inglaterra como nos Estados Unidos estava pouco profissionalizado e que novos estudos deveriam ser investigados.

Price permaneceu em Cambridge por dois anos depois de concluir seu segundo doutorado em 1954; após uma nomeação de um ano no Smithsonian Institution, Washington, DC, Price retornou a Princeton em 1958 para desenvolver estudos durante dois anos no Instituto de Estudos Avançados. Nos meses de outubro e novembro de 1959, a convite do Departamento de História da Universidade de Yale, Price fez cinco palestras públicas na Sterling Memorial Biblioteca em New Haven. A combinação de textos das palestras, incluindo uma intitulada Diseases of science, "expandiu ainda mais sobre o tema do crescimento exponencial e que foi publicado posteriormente no livro Science since Babylon, em 1961” (FURNER, 2003a, p.116).

Em 1960, Price foi nomeado professor de História da Ciência no novo Departamento de História da Ciência e da Medicina criado por John Fulton em Yale, tornando-se chefe do departamento no ano seguinte, e lá permanecendo lá até sua morte em 1983. Em junho de 1962, 
como o quarto detentor anual do George B. Pegram Lectureship em Brookhaven National Laboratory, NY, Price ministrou quatro palestras naquele centro de pesquisa atômica, e estas foram igualmente recolhidas no volume publicado no ano seguinte como Little science, big science (PRICE, 1963a). Em cada uma dessas palestras Price desenvolveu uma seleção diferente das ideias discutidas anteriormente em Diseases of science (FURNER, 2003a; YAGI, BADASHI, BEAVER, 1996; TURNER, 1984; KOCHEN, 1984).

Nos anos 1970, já totalmente à vontade nos estudos relativos aos aspectos sociais da ciência, Price assumiu ao lado de Eugene Garfield e outros membros mais proeminentes do "colégio invisível" dos cientometristas, funções honorárias ou ativas no Conselho Editorial do periódico Scientometrics, hoje considerado um dos mais conceituados periódicos científicos relacionados ao campo dos estudos métricos da informação, e que havia sido lançado em 1978 como uma joint venture entre a Elsevier Science Publishing Company, Amsterdã e a Akadémiai Kiadó, em Budapeste (SCHUBERT, 2001, p. 183).

Como refere Griffith (1984, p. 5) "as conquistas de Price ao analisar as dimensões sociais da ciência foram extremamente ricas e variadas". Ao criar um modelo estatístico coerente para a ciência, constantemente refinado e ampliado, seus estudos foram fundamentais para exigir preocupação simultânea com as características históricas, sociais e analíticas de cada problema de pesquisa.

Na visão de Yagi; Badash e Beaver (1996, p. 64), as descobertas de Price sobre o crescimento exponencial da ciência, cientistas e artigos científicos foram apenas o começo de uma série de contribuições fundamentais para os indicadores de ciência e a modelagem quantitativa da atividade científica, que incluiu "o uso de citações para analisar e mapear campos científicos juntamente com suas estruturas e evolução ao longo do tempo, distribuições e estruturas de autorias e teorias de vantagem cumulativa e a colaboração informal nos colégios invisíveis, entre outros".

No Brasil, a influência dos estudos de Price se faz notar desde os anos 1970, com a implantação do Programa de Mestrado em Ciência da Informação ,no Instituto Brasileiro de Informação em Ciência e Tecnologia (IBICT). Essa instituição foi pioneira nos estudos bibliométricos e cientométricos realizados no país, e naquela ocasião, Tefko Saracevic, Bert Roy Boyce e Wilfred Lancaster, entre outros, introduziram os métodos bibliométricos em disciplinas 
ministradas. Esses pesquisadores assumiram as primeiras orientações com essas abordagens, entre outras as realizadas por Gilda Maria Braga, Laura Maia de Figueiredo e Elsa de Lima e Silva Maia no ano de 1973, conforme relatam Urbizagástegui-Alvarado (1984) e Pinheiro e Loureiro (1995). Entre essas pesquisas, o estudo de Braga (1973) intitulado Relações bibliométricas entre a frente de pesquisa (Research Front) e revisões da literatura: estudo aplicado a Ciência da Informação, foi pioneiro por abordar o conceito de frente de pesquisa elaborado pelo físico e historiador da ciência Derek John de Solla Price. Um ano mais tarde, outro estudo de Braga (1974) sintetizou o pensamento de Price consolidado em diversas publicações. Pinheiro e Loureiro (1995, p.11) relatam que "Price manteve contatos constantes com pesquisadores brasileiros de Ciência da Informação, notadamente Gilda Braga”. Ainda nos anos 1970, a revista Ciência da Informação publicou três artigos de Price (1974) e Price e Gürsey (1975a; 1975b).

No Brasil, Price também ministrou conferências, entre elas no Seminário Internacional de Estudos sobre Política Científica, ocorrido em 1978 no Rio de Janeiro, e intitulada Ciência da Ciência: uma contribuição para a política científica brasileira Nessa ocasião, Price relata que esteve no Brasil três vezes, a primeira como turista, a segunda vez em 1975, e "depois de um período de pesquisas e palestras fiz um relatório sumário sobre política cientifica no Brasil [...] e propus a criação de uma pequena unidade de política científica no CNPq. (PRICE, 1978a, p.38).

\section{A PRESENÇA DE DEREK DE SOLLA PRICE NOS ARTIGOS DA COLEÇÃo SciELO.br}

Para o desenvolvimento dessa pesquisa optou-se como fonte de dados a biblioteca eletrônica SciELO.br por abranger uma coleção de 294 títulos de periódicos científicos de várias áreas de conhecimento e de grande visibilidade na produção científica brasileira. Para os procedimentos adotados foram realizadas as seguintes etapas: a) a coleta de dados foi realizada no dia 12 de fevereiro de 2020 por meio do formulário básico buscando nos campos pesquisar e todos os índices, de forma individualizada e sem delimitação de tempo, as seguintes palavras-chave que resultaram em: bibliometria $(\mathrm{n}=240)$, bibliométrico $(\mathrm{n}=157)$, bibliométrica, $(\mathrm{n}=166)$, cientometria $(n=52)$, cientométrica $(n=9)$, cientométrico $(n=9)$; b) os dados recuperados foram transferidos para o software Excel para descrição e categorização; c) em seguida, os registros repetidos $(\mathrm{n}=242)$ 
foram eliminados; d) as referências dos artigos remanescentes $(n=391)$ foram analisadas visando identificar aqueles que citaram obras de Price (artigos, livros, capítulos, trabalhos em eventos). Vale observar que foram descartados muitos artigos $(n=361)$ que não citaram Price, e outros $(n=2)$ que apesar de citarem Price no corpo do texto, não incluíram esse autor nas referências. Desse modo, após a análise de citações obteve-se o total de artigos que compuseram corpus final da pesquisa $(n=27)$.

A Figura 2 apresenta a distribuição dos artigos ao longo de 21 anos. Observa-se que os primeiros artigos datam do ano de 1998 e o mais recente é de 2019. Calculada a média de artigos $(n=1,42)$ por ano pode ser considerada baixa, haja vista a importância do autor no campo dos estudos métricos da informação, e dado que os estudos bibliométricos chegaram no Brasil na década de 1970 .

Figura 2 - Evolução temporal dos artigos da coleção SciELO.br que citaram Price

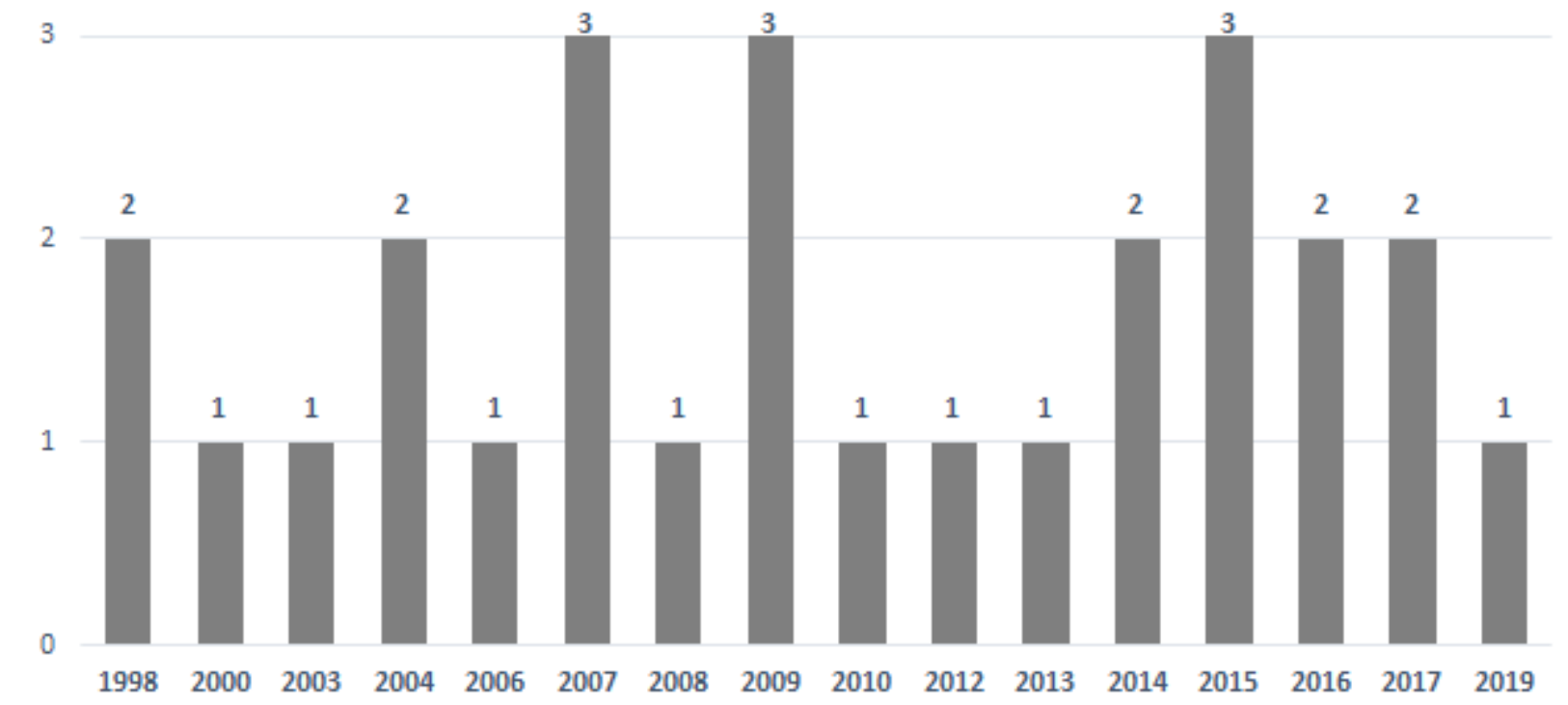

Fonte: Elaborado pelos autores

Pode-se supor que essa baixa quantidade de artigos que citaram as obras de Price deve-se a fatores que provavelmente estão interligados, tais como: a dificuldade de obtenção de alguns textos integrais de Price, a despeito da disponibilidade da literatura científica internacional na Internet; a barreira linguística, pois a maior parte dos textos que foram publicados por Price não foram traduzidos para a língua portuguesa, com exceção, por exemplo dos livros Big Science, Little Science e Science since Babylon. Em vista disso, acredita-se que Price passou a ser citado nos 
artigos de autores brasileiros por meio do recurso do "apud", ou seja, por meio daqueles que tiveram acesso aos textos integrais de Price,

Uma outra possibilidade da ausência de artigos que citaram as obras de Price pode estar relacionada às matrizes curriculares dos cursos de graduação e pós-graduação no Brasil, uma vez que são poucas as disciplinas relativas ao campo dos estudos métricos da informação ofertadas em programas ou cursos de graduação e pós-graduação tanto da área de Ciência da Informação quanto de outros campos de conhecimento.

Os dois primeiros artigos que citaram Price em suas pesquisas foram publicados na revista Ciência da Informação no ano de 1998. O primeiro trabalho intitulado "O papel da informetria e da cienciometria e sua perspectiva nacional e internacional", de autoria de Cesar Macias-Chapula, aborda o conceito de frente de pesquisa ao citar a obra Little science, big Science, publicada em 1963. O segundo artigo, de autoria de Lídia Alvarenga com o título de "Bibliometria e arqueologia do saber de Michel Foucault: traços de identidade teórico-metodológica” citou o artigo de Price Networks of scientific papers publicado em 1965 para explicar as redes de colaboração científica e o conceito de bibliometria.

Ao investigar as autorias dos artigos $(n=27)$, os resultados mostraram um total $(n=49)$ de diferentes autores e coautores, reunindo artigos em coautoria $(n=16)$ e individuais $(n=11)$. A maioria dos autores ( $n=30)$ é do campo da Ciência da Informação, seguidos pelos da Administração $(\mathrm{n}=5)$, da Fonoaudiologia $(\mathrm{n}=3)$. Os autores das áreas de Medicina $(\mathrm{n}=2)$, Ciências Biológicas $(n=2)$, Odontologia $(n=2)$, Sociologia da Ciência $(n=2)$, Psicologia $(n=1)$, Ciências Sociais $(n=1)$ e Economia $(n=1)$ tiveram a menor representatividade. Não chega a ser surpresa que os autores da área de Ciência da Informação sejam majoritários, uma vez que esta pode ser considerada como a pioneira nos estudos métricos da informação no Brasil.

As citações das obras de Price $(n=38)$ nos artigos analisados $(n=27)$, variaram entre quatro obras citadas em um artigo; três obras, citadas em três artigos, e duas obras citadas em dois artigos, sendo que nos demais artigos $(n=21)$ apenas uma obra de Price foi citada.

Apesar de Price ter deixado um legado de mais de três centenas documentos, entre livros, capítulos de livros, artigos, e trabalhos em eventos, e outros, apenas 15 obras do autor - incluindo duas traduções para o português - foram citadas nos artigos que adotaram abordagens InCID: R. Ci. Inf. e Doc., Ribeirão Preto, v. 12, n. 1, p. 19-40, mar./ago. 2021. 
bibliométricas e cientométricas presentes na coleção de periódicos da biblioteca eletrônica SciELO $(\mathrm{n}=27)$. A Tabela 1 apresenta os títulos das obras com seus respectivos anos de publicação, e o total de citações recebidas nos artigos.

Tabela 1 - Obras de Price citadas nos artigos

\begin{tabular}{l|c}
\hline \multicolumn{1}{c|}{ Títulos das obras / Ano de publicação } & Citações \\
\hline Little Science, Big Science (1963a) & 10 \\
O desenvolvimento da ciência* (1976b) & 9 \\
\hline Networks of scientific paper (1965) & 5 \\
\hline The structures of publication in science and technology (1969a) & 3 \\
\hline Collaboration in an invisible college (1966) & 2 \\
\hline Litle science, big science... and beyond (1986) & 2 \\
\hline Science since Babylon (1961) & 1 \\
A ciência desde a Babilônia* (1976c) & 1 \\
\hline Toward a model for science indicators (1978b) & 2 \\
\hline A calculus of science (1963b) & 1 \\
\hline Editorial statements (1978c) & 1 \\
\hline Measuring the size of science (1969b) & 1 \\
\hline Society's needs in scientific and technical information (1975) & 1 \\
\hline Some remarks on elitism in information and the invisible college & 1 \\
phenomenon in science (1971a) & 1 \\
\hline The expansion of scientific knowledge (1971b) & $\mathbf{4 1 * *}$ \\
\hline
\end{tabular}

(*) Traduções para o português dos livros Little science, big science e Science since Babylon (**) cada artigo pode ter citado mais do que uma obra de Price

Fonte: Elaborado pelos autores

A obra mais citada nos artigos que compuseram o corpus dessa pesquisa $(n=27)$ foi o livro O desenvolvimento da ciência (Little science, big science), com 21 citações, quando somadas as edições em português e inglês. Ao examinar a obra Little Science big science (LSBS), Furner (2003a, p. 115) argumenta que as ideias de Price,

[...] foram formuladas durante um período crucial no desenvolvimento de estudos sóciohistóricos da ciência. O talento do Price para inovação e síntese em um ambiente tempo altamente carregado, e sua apreciação do trabalho pioneiro em estudos de ciência do cristalógrafo J.D. Bernal, refletem-se nos aspectos inequivocamente profundos e abrangentes nos quais LSBS contribuiu para o desenvolvimento da teoria cientométrica e sociológica.

Furner (2003a; 2003b) e Michaelis (1993) complementam que nesse mesmo livro Price (1963a) desenvolveu a base estatística de conceitos como Big Science e de colégio invisível. Price 
também introduziu a observação de que 80 a $90 \%$ de todos os cientistas que já viveram estão vivos agora, e discutiu a mudança fundamental da pesquisa científica de pequena escala por um único pesquisador para os esforços da grande equipe que se tornaram tão comuns em muitas disciplinas durante o período de 30 anos entre as décadas de 1930 e 1960.

Já Martín-Martín; Orduna-Malea e López-Cózar (2018, p. 1.261), detectaram que entre os 25 documentos mais influentes no campo da bibliometria, Price tem dois textos, sendo que o livro Little Science, Big Sicence (PRICE, 1963a) é o mais influente nos estudos métricos de acordo com as citações ( $\mathrm{n}=5.410)$ no Google Acadêmico, bem como o artigo A general theory of bibliometric and other cumulative advantage processes (PRICE, 1976a) que é o $17^{\circ}$ mais citado, com 1.148 citações.

O segundo texto mais citado pela comunidade científica dos estudos métricos da informação foi o artigo Network of scientific paper, em que Price (1965) introduz uma discussão acerca das noções sobre redes de ciência. Moed (2008), discorre que os mapeamentos atuais da estrutura e desenvolvimento da atividades científicas e identificação das redes de artigos, de pesquisadores individuais e grupos, são todos baseados nesse documento. Ronda-Pupo e Pham (2018, p. 366) vão além ao afirmarem que este artigo introduziu as noções de redes de ciência nos estudos cientométricos e "pode ser considerado um dos documentos fundantes da atual disciplina Cientometria”.

A Tabela 2 mostra os vários periódicos $(n=13)$ que publicaram os artigos que citaram Price.

Tabela 2 - Periódicos e respectivas áreas que publicaram os artigos com citações de Price

\begin{tabular}{l|l|c}
\hline Título dos Periódicos & \multicolumn{1}{c|}{ Áreas } & Artigos \\
\hline Ciência da Informação & \multirow{2}{*}{ Ciência da Informação } & 9 \\
\cline { 3 - 3 } Transinformação & & 5 \\
\cline { 1 - 1 } Perspectivas em Ciência da Informação & Ciências & 3 \\
\hline Anais da Academia Brasileira de Ciências & Educação em Ciências & 1 \\
\hline Ciência \& Educação (Bauru) & Saúde Coletiva & 1 \\
\hline Ciência \& Saúde Coletiva & $\begin{array}{l}\text { História das Ciências e da } \\
\text { Saúde }\end{array}$ & 1 \\
\hline História, Ciências, Saúde-Manguinhos & Fonoaudiologia & 1 \\
\hline Pró-Fono: Revista de Atualização Científica & Psicologia & 1 \\
\hline Psico-USF & Administração & 1 \\
\hline RAM-Revista de Administração Mackenzie & Administração & 1 \\
\hline Revista de Administração Contemporânea & Administração & 1 \\
\hline REAd-Revista Eletrônica de Administração & &
\end{tabular}

InCID: R. Ci. Inf. e Doc., Ribeirão Preto, v. 12, n. 1, p. 19-40, mar./ago. 2021. 


\begin{tabular}{c|c|c}
\hline Revista Brasileira de Educação Médica & Saúde Pública & 1 \\
\hline \multicolumn{2}{c|}{ Total } & $\mathbf{2 7}$ \\
\hline
\end{tabular}

Fonte: Elaborado pelos autores

O periódico com mais publicações $(\mathrm{n}=9)$ é Ciência da Informação, uma das mais antigas e tradicionais revistas brasileiras no campo Ciência da Informação no Brasil, e pioneira na publicação de estudos bibliométricos que utilizaram conceitos de Price em seus artigos (BRAGA, 1973, 1974; CARVALHO, 1975; SÁ, 1976; PARANHOS, 1976). Inclusive, conforme já comentado, esse periódico já publicou alguns textos do próprio autor (PRICE, 1974; PRICE; GÜRSEY, 1975a; 1975b), ou seja, essas evidências podem ser um indicativo de que os estudos bibliométricos têm boa receptividade nesse periódico, estimulando outros pesquisadores a publicarem nessa revista.

Destaca-se ainda que a somatória dos artigos $(n=17)$ dos periódicos da área de Ciência da Informação foram responsáveis por $63 \%$ do total de artigos citados $(n=27)$. Tais periódicos denotam que essa área do conhecimento vai ao encontro do que Larivière (2012, p. 13) afirmou sobre o fato de que "os métodos bibliométricos estão no coração da Ciência da Informação". Em relação ao escopo de interesse dos periódicos de outras áreas $(n=8)$ observa-se que esses também abrem espaço para pesquisas bibliométricas e cientométricas apoiadas em obras de Price, sugerindo que esse autor também é uma referência em pesquisas de outros campos do conhecimento.

Na pesquisa aqui relatada, também foi adotada a metodologia da análise de conteúdo. Os $\operatorname{artigos}(n=27)$ foram lidos integralmente, permitindo identificar quais os conceitos $(n=9)$ presentes nas obras de Price e suas aplicações $(n=12)$ foram utilizadas nesses trabalhos (Tabela 3)

Tabela 3 - Conceitos e aplicações de Price utilizadas nos artigos

\begin{tabular}{l|l|c}
\hline \multicolumn{1}{c|}{ Conceitos } & \multicolumn{1}{|c}{ Aplicações } & Artigos \\
\hline Little Science & Desenvolvimento da ciência & 2 \\
Big Science & Crescimento da literatura científica & 4 \\
Crescimento exponencial da & & 3 \\
ciência & Colaboração científica & 5 \\
\hline \multirow{2}{*}{ Colégio invisível } & Redes científicas & 3 \\
\hline & Produtividade científica & 3 \\
Lei do Elitismo & Produtividade de autores & 5 \\
Frente de pesquisa & Análise de citações & 4 \\
& Análise de autoria e coautorias & 2 \\
\hline Bibliometria & Indicadores bibliométricos & 5 \\
Cientometria & Indicadores cientométricos & 2 \\
Política científica & Indicadores de impacto & 1 \\
\hline
\end{tabular}

InCID: R. Ci. Inf. e Doc., Ribeirão Preto, v. 12, n. 1, p. 19-40, mar./ago. 2021. 


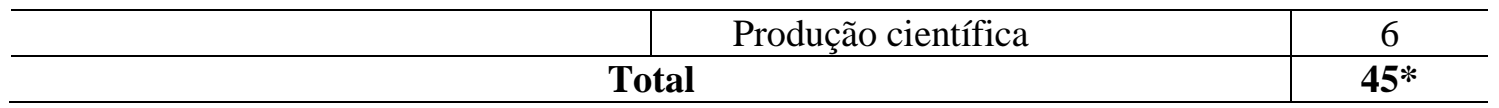

(*) Cada artigo pode ter utilizado mais de um conceito ou aplicação

Fonte: Elaborado pelos autores

Os conceitos de Bibliometria, Cientometria, e Política Científica e suas aplicações foram os mais utilizados nos artigos $(\mathrm{n}=14)$. Apesar do termo Cientometria ter surgido na antiga União Soviética e Europa Ocidental, foi Price que na década de 1960 impulsionou esse campo de investigação ao mostrar que a Cientometria é uma disciplina científica que estuda as estruturas e as propriedades da informação científica. A Cientometria vai mais além do que a Bibliometria e suas técnicas para estudar a produção científica, ou seja, ao produzir indicadores que servem para orientar e desenvolver e comparar políticas científicas de países, regiões, analisando seus aspectos econômicos e sociais. (SPINAK, 1996). Além disso, as aplicações da Bibliometria e Cientometria podem se traduzir em indicadores de produção científica, tecnológica e de impacto.

Além disso, a produtividade científica e de autores, bem como a análise de citações, de autoria e coautorias foram aplicações presentes em artigos $(\mathrm{n}=14)$ advindas dos conceitos da Lei do Elitismo e Frente de Pesquisa formulados por Price. Vale lembrar que ele propôs estudar a produção científica de pesquisadores pelos métodos da própria ciência, e para isso recorreu às contribuições de Lotka formulada em 1926, concluindo que 1/3 da literatura é produzida por menos de 1/10 dos autores mais produtivos, levando a uma média de 3,5 documentos por autor, com $60 \%$ dos autores produzindo um único documento. Dessa forma, Price (1976a) propôs a Lei do Elitismo por meio de uma expressão matemática em que $n$ representa o número total de contribuintes em uma disciplina e o cálculo da $\sqrt{ } n$ permite identificar a elite da área estudada, de Frente de Pesquisa formulado por Price (1965) refere-se aos artigos mais citados da literatura recente, os quais, "a qualquer momento podem representar o estado da arte de um campo científico”. (ZUPIC; CATER, 2015, p. 439).

Já o conceito de Big science - normalmente associado à grande ciência que foi alimentada pelos governos e pelas enormes instalações de pesquisa, após a 2a . Guerra Mundial -, e o de Little Science-geralmente produzida por pesquisadores que trabalham sozinhos ou em pequenos grupos; de crescimento exponencial da ciência - explicado pelo fato de que a taxa de crescimento da população científica é de tal ordem que o tamanho da ciência duplicou a cada dez ou 15 anos desde o século XVII -, foram utilizados nos artigos $(n=9)$ para explicar o desenvolvimento da ciência e o 
crescimento da literatura científica. Esses conceitos estão presentes principalmente nos livros Science Since Babylon (PRICE, 1961) e Big Science, Little Science (PRICE, 1963a), e Big Science, Little Science... and beyond (PRICE, 1986).

Por sua vez, o conceito de Colégio Invisível refere-se ao conjunto de pesquisadores que compartilham um interesse comum e se comunicam entre eles, embora não formem parte de uma instituição formal (PRICE; BEAVER, 1966; PRICE, 1971b), e suas aplicações em estudos de colaboração científica e redes científicas foram identificados no menor volume de artigos $(\mathrm{n}=8)$ que utilizaram esse constructo teórico.

Como referiu Merton (1973, p. 507), Derek de Solla Price sugeriu vários recursos que distinguem as literaturas "das ciências hard - exatas e tecnológicas, daquelas soft, de outros campos de conhecimento".

\section{Considerações finais}

Os dados da pesquisa revelaram que apesar do considerável volume de artigos recuperados $(\mathrm{n}=391)$ na biblioteca eletrônica SciELO.br e que adotaram as abordagens bibliométricas e cientométricas, somente $6,9 \%(n=27)$ citaram as obras de Derek John de Solla Price em suas pesquisas. A despeito disso, a análise de citações revelou que os autores do campo da Ciência da Informação são predominantes quando se trata de citar as obras de Price.

Entre as citações de Price, destaca-se Little Science, Big Science como a obra que os autores dos artigos mais recorreram para fundamentar suas pesquisas. Contudo, apesar da primeira edição desta obra traduzida no Brasil ser datada de 1976, os dois primeiros artigos que citaram esse livro são de 1998, ou seja, 22 anos da publicação dessa edição brasileira e 35 anos após a primeira edição na língua inglesa.

Vale observar que diversos artigos que foram descartados na pesquisa citaram Price pela via do "apud" sem ter recorrido as suas obras originais, e ao contrário, valendo-se de outros escritos que citam esse autor. Esse comportamento de citação sugere que os estudos clássicos de Price não são lidos integralmente e são substituídos por artigos e obras elaborados por comentadores ou leitores dos trabalhos originais. Aliado a isso, artigos que adotaram as abordagens bibliométrica e 
cientométrica, mas não incluíram as palavras-chave utilizadas nessa pesquisa, e ainda periódicos que não estão com a coleção completa disponível na SciELO.br, também ficaram de fora da pesquisa realizada.

O estudo comprovou ainda que os conceitos de Bibliometria e Cientometria, advindos das obras desse que é considerado um dos fundadores desse campo científico, foram os mais citados nos artigos que utilizaram métricas científicas para analisar a literatura científica e/ou produzir indicadores de ciência e tecnologia.

Imperioso destacar que a vasta produção científica de Price e os conceitos nela amplamente expostos e debatidos vão além das obras citadas $(n=13)$ ou dos conceitos $(n=8)$ identificados nos achados desse manuscrito, ou seja, há um conjunto de artigos, editoriais, livros e temáticas publicados por Price que também podem ser explorados pelos pesquisadores do campo da Bibliometria e Cientometria.

Em que pese o fato de que os resultados obtidos estejam aquém do volume e importância da obra de Price e de suas contribuições para os estudos métricos da ciência, ainda assim não é possível afirmar que há uma negligência dos pesquisadores dessa área na utilização dos conceitos de Price e suas aplicações, uma vez que a biblioteca eletrônica SciELO.br não indexa todos os periódicos brasileiros. Assim, recomenda-se que em trabalhos futuros sejam utilizadas outras fontes de dados para ampliar os achados do estudo aqui relatado.

À guisa de conclusão, vale encerrar a pesquisa realizada corroborando o argumento de Fernández-Cano, Torralbo e Vallejo (2004, p.301), ao afirmarem que Derek John de Solla Price, "sem dúvida, representa um gigante com grandes ombros onde nós, as pessoas comuns, buscamos apoio. Price, principal líder intelectual, nos ofereceu um paradigma para o estudo da ciência em geral e de suas várias disciplinas ou ciências em particular". 


\section{Referências}

ADEDAYO A. V. Prizing de Solla Price's circumvent. Elixir Library Science, [s. l.], v. 83, p. 32849-32850, 2015.

ALVARENGA, L. Bibliometria e arqueologia do saber de Michel Foucault: traços de identidade teórico-metodológica. Ciência da Informação, Rio de Janeiro, v. 27, n. 3, 1998.

BEDINI, S. Éloge: Derek de Solla Price (1922-1983). Annali dell'Istituto e Museo di Storia della Scienza di Firenze, Firenze, v. 9, p. 95-115, 1984.

BRAGA, G. M. Relações bibliométricas entre a frente de pesquisa (research front) e revisões de literatura: estudo aplicado à Ciência da Informação. Ciência da Informação, Rio de Janeiro, v. 2, n. 1, p. 9-26, 1973.

BRAGA, G. M. Informação, ciência, política científica: o pensamento de Derek de Solla Price. Ciência da Informação, Rio de Janeiro, v. 3, n. 2, p. 155-177, 1974.

CARVALHO, M. L. B. Estudo de citações da literatura produzida pelos professores do Instituto de Ciências Biológicas da UFMG. Ciência da Informação, Rio de Janeiro, v. 5, n. 1/2, p. 27-42, 1975.

BONITZ, M. The multidimensional space of scientometrics: the Derek de Solla Price Medal. Scientometrics, [s. l.], v. 29, n. 1, p. 3-14, 1994.

BUCKLAND, M. Some european contributions to Information Science. Bulletin of the Association for Information Science \& Technology, [s. l.], v. 43, n. 3, p. 49-53, 2017.

CHAVARRO, D. Exploring research evaluation from a sustainable development perspective. In: KRAEMER-MBULA, E. (ed.) et al. Transforming research excellence: new ideas from the Global South. South Africa: African Minds, 2020.

CRAWFORD, S. Derek de Solla Price (1922-1983): the man and the contribution. Bulletin of the Medical Library Association, [s. l.], v. 72, n. 22, 1984.

ERFANMANESH, M.; MOGHISEH, Z. How winning an international scientifc award affects publishing behavior of laureates: the case of Derek de Solla Price Medal in Scientometrics. Publishing Research Quarterly, [s. l.], v. 35, n. 2, p. 201-202, jun. 2019.

FERNÁNDEZ-CANO, A.; TORRALBO, M.; VALLEJO, M. Reconsidering Price.s model of scientific growth: an overview. Scientometrics, [s. l.], v. 61, n. 3, p. 301-321, 2004.

FIGUEIREDO, L. M. Distribuição da literatura geológica brasileira: estudo bibliométrico. Ciência da Informação, Rio de Janeiro, v. 2, n. 1, p. 27-40, 1973. 
FURNER, J. Little book, big book: before and after Little science, big science: a review article, Part I. Journal of Librarianship and Information Science, [s. l.], v. 35, n. 2, p. 115-125, 2003a.

FURNER, J. Little book, big book: before and after Little science, big science: a review article, Part II. Journal of Librarianship and Information Science, [s. l.], v. 35, n. 2, p. 189-201, 2003b.

GALYAVIEVA, M. S. Bibliometric analysis of the document flow of informetrics based on the Russian Science Citation Index. Scientific and Technical Information Processing, [s. l.], v. 41, n. 4, p. 220-229, 2014.

GARFIELD, E. From the science of science to scientometrics visualizing the history of science with HistCite software. Journal of Informetrics, [s. l.], v. 3, n. 3, p. 173-179, 2009.

GARFIELD, E. Celebrating the legacy of de Solla Price. Research Trends, [s. l.], v. 7, 2008.

GARFIELD, E. A tribute to Derek John de Solla Price: a citation analysis of little science, big sicence. Scientometrics, [s. l.], v. 7, n. 3-6, 1985.

GINGRAS, Y. Os desvios da avaliação da pesquisa: o bom uso da bibliometria. Rio de Janeiro: Ed. UFRJ, 2016.

GRIFFITH, B. C. Derek Price (1922-1983) and the social studies of science. Scientometrics, [s. l.], v. 6, n. 1, p. 5-7, 1984.

HAYASHI, M. C. P. I. Afinidades eletivas entre a cientometria e os estudos sociais da ciência. Filosofia e Educação, Campinas, v. 5. n. 2, p. 57-88, out. 2013.

International Society for Scientometrics and Informetrics (ISSI). Derek de Solla Price Memorial Medal. 2020. Disponível em: http://www.issi-society.org/awards/derek-de-sollaprice-memorial-medal/. Acesso em: 07 abr. 2020.

KINOUCHI, R. R. Scientometrics: the project for a science of science transformed into an industry of measurements. Scientiae Studia, São Paulo, v. 12, p. 147-59, 2014.

KOCHEN, M. Toward a paradigma for Information Science: the influence of Derek de Solla Price. Journal of the American Society for Information Science, [s. l.], v. 35, n. 3, p. 147-148, 1984.

LARIVIÈRE, V. The decades of metrics? Examining the evolution of metrics within and outside LIS. Bulletin fo the American Society for Information Science and Sechnology, [s. l.], v. 38, n. 6, p. 13-17, 2012.

MACIAS-CHAPULA, C. O papel da informetria e da cienciometria e sua perspectiva nacional e internacional. Ciência da Informação, Rio de Janeiro, v. 27, n. 2, p. 134-140, maio/ago. 1998. 
MAIA, E. L. S. Comportamento bibliométrico da língua portuguesa como veículo da representação da informação. Ciência da Informação, Rio de Janeiro, v. 2, n. 2, p. 99-138, 1973.

MARTÍN-MARTÍN, A.; ORDUNA-MALEA, E.; LÓPEZ-CÓZAR, E. D. A novel method for depicting academic disciplines through Google Scholar Citations: the case of bibliometrics.

Scientometrics, [s. l.], v. 114, p. 1251-1273, 2018.

MATHIAS, S. Apresentação da edição brasileira. In: PRICE, D. J. S. O desenvolvimento da ciência. Rio de Janeiro: LTC, 1976.

MERTON, R. K. The sociology of science: theoretical and empirical investigations. Chicago: The University of Chicago Press, 1973.

MICHAELIS, A. R. Interdisciplinary big science. Interdisciplinary Science Reviews, [s. l.], v. 18, n. 3, p. 177-179, Sep. 1993.

MOED, H. K. Why the citation cycle is my favorite de Solla Price paper. Research Trends, $[s$. l.], v. 7, Sep. 2008.

MOKHTARPOUR, R.; KHASSEH, A. A. Who is who in library and information science research?: the integrative application of scholarly influence indicators. Journal of Librarianship and Information Science, [s. l.], Feb. 2020.

PARANHOS, W. M. M. R. Análise do banco de dados do IBBD: atividades de pesquisa em Química no Brasil, relativas a 1973. Ciência da Informação, Rio de Janeiro, v. 5, n. 1/2, p. 1726, 1976.

PINHEIRO, L. V. R.; LOUREIRO, J. M. M. Traçados e limites da Ciência da Informação. Ciência da Informação, Rio de Janeiro, v. 24, n. 1, 1995.

PRICE, D. S. Science since babylon. New Haven: Yale University Press, 1961.

PRICE, D. S. Little science, big science. New York: Columbia University Press, 1963a.

PRICE, D. S. A calculus of science. International Science and Technology, [s. l.], v. 15, p. 37 43, Mar. 1963b.

PRICE, D. S. Networks of scientifc papers: the pattern of bibliographic references indicates the nature of the scientific research front. Science, [s. l.], v. 149, n. 3683, p. 510-515, Jul. 1965.

PRICE, D. S.; BEAVER, D. B. Collaboration in an invisible college. American Psychologist, [s. l.], v. 21, p. 1011-1018, 1966.

PRICE, D. S. The structures of publication in science and technology. In: GRUBER, William H.; 
MARQUIS, Donald R. Factors in the transfer of technology. Cambridge: MIT Press, 1969a. p. 179-180.

PRICE, D. S. Measuring the size of science. Proceedings of the Israel Academy of Sciences and Humanities, [s. l.], v. 4, n. 6, p. 98-111, $1969 \mathrm{~b}$.

PRICE, D. S. Some remarks on elitism in information and the invisible college phenomenon in science. Journal of the American Society for Information Science, [s. l.], v. 22, p. 74-75, 1971a.

PRICE, D. S. The expansion of scientific knowledge. Annals of the New York Academy of Sciences, [s. l.], v. 184, p. 257-259, June 1971b.

PRICE, D. S. Society's needs in scientific and technical information. Ciência da Informação, Brasília, v. 3, p. 97-103, 1974.

PRICE, D. S.; GÜRSEY, S. Studies in scientometrics. Part I. transience and continuance in scientific authorship. Ciência da Informação, Brasília, v. 4, n. 1, p. 27-40, 1975a.

PRICE, D. S.; GÜRSEY, S. Studies in scientometrics. Part II. the relation between source author and cited author populations. Ciência da Informação, Brasília, v. 4, n. 2, p. 103-108, 1975 b.

PRICE, D. S. A general theory of bibliometric and other cumulative advantage processes. Journal of the American Society for Information Science, [s. l.], v. 27, p. 292-306, 1976a.

PRICE, D. S. O desenvolvimento da ciência: análise histórica, filosófica, sociológica e econômica. Trad. de Simão Mathias com a colaboração de Gilda Braga. Rio de Janeiro: Livros Técnicos e Científicos, $1976 b$.

PRICE, D. S. A ciência desde a Babilônia. Trad. de Leônidas Hegenberg e Octanny S. da Mota. Belo Horizonte: Itatiaia, 1976c.

PRICE, D. S. Ciência da ciência: uma contribuição para a política científica brasileira. In:

Seminário Internacional de Estudos sobre Política Científica, 1978. Anais..., Rio de Janeiro, 6 a 10 de março 1978a. p. 37-50.

PRICE, D. S. Toward a model for science indicators. In: ELKANA, Y. et al. Toward a metric of science: the advent of science indicators. New York: John Wiley \& Sons, 1978b. p. 69-95.

PRICE, D. S. Editorial statements. Scientometrics, [s. l.], v. 1, p. 7-8, 1978c.

PRICE, D. S. Little science, big science... and beyond. New York: Columbia Press, 1986.

RONDA-PUPO, G. A.; PHAM, T. The evolutions of the rich get richer and the fit get richer phenomena in scholarly networks: the case of the strategic management jornal. Scientometrics, [s.l.], v. 116, p. 363-383, 2018. 
SÁ, E. S. Participação dos pesquisadores de microbiologia, imunologia e parasitologia (MIP) na literatura científica internacional. Ciência da Informação, Rio de Janeiro, v. 5, n. 1/2, p. 43-69, 1976.

SCHUBERT, A. Scientometrics: the research field and its journal. In: HECK, A. (ed.). Organizations and strategies in Astronomy II. Dordrecht: Kluwer Academic, 2001. p. 179195.

SPINAK, E. Diccionario enciclopédico de bibliometría, cienciometría e informetría. [S. $l$.]: UNESCO, 1996.

TURNER, G. L. Obituary Derek John de Solla Price 1922-1983. Annals of science, [s. l.], v. 41, p. $105-107,1984$.

URBIZAGÁSTEGUI-ALVARADO, R. A bibliometria no Brasil. Ciência da Informação, Brasília, v. 13, n. 12, p. 91-105, jul./dez. 1984.

VAN NOORDEN, R. A profusion of measures. Nature, [s. l.], v. 465, p. 864-866, 17 June 2010.

WOUTERS, P.; LEYDESDORFF, L. Has Price's dream come true: is scientometrics a hard science? Scientometrics, [s. l.], v. 31, n. 2, p. 193-222, 1994.

YAGI, E.; BADASH, L.; BEAVER, D. B. Derek J. de S. Price (1922-83): historian of science and herald of scientometrics. Interdisciplinary Science Reviews, [s. l.], v. 21, n. 1, p. 64-84, 1996.

ZUPIC, I.; CATER, T. Bibliometric methods in management and organization. Organizational Research Methods, [s. l.], v. 18, n. 3, p. 429-432, 2015. 